\title{
Grand challenges in computational physics
}

\section{Christian Klingenberg*}

Department of Mathematics, Mathematical Fluid Mechanics, Universität Würzburg, Würzburg, Germany

*Correspondence: klingen@mathematik.uni-wuerzburg.de

\section{Edited by:}

Alex Hansen, Norwegian University of Science and Technology, Norway

\section{INTRODUCTION: EXPERIMENT AND THEORY}

To put things into perspective, we step back for a minute, and remember what the overarching goal of science is. It is to gain insight into a given natural phenomena (NP). Traditionally there are two steps to achieve this.

The first step (we shall call experiment) is observation and experiments. They give sets of data we have of the NP at hand. This is the concrete manifestation of the NP. The second step (we shall call theory) is to devise a theory that is an idealized model of the NP. Theory is typically expressed in the language of mathematics and needs mathematical theory, more recently also theoretical computer science. The quality of of a theoretical mathematical model depends on how far along mathematical and computer science theory has come.

\section{COMPUTATIONAL SCIENCE AS THE THIRD MODE}

Since the NP can be grasped only through the data obtained in the experiment, this data needs to be connected to its theory. Scientific computation will take the mathematical model and compute its ramification for the situation in which the data had been gathered. This is the classical role of computation: validation of theory through computation. Traditionally validation is done by experiment, but computation is gaining an ever increasing role in validation. For example, when studying fluid mixing, the theories on chaotic mixing are validated by both experiments and detailed computations. Using computations, the latter allows for parameter studies not possible with experimental setups. Assuring that these computations mimic the NP that is being studied is a big challenge.

In Valdes-Perez (1993) proclaimed the paradigm of Computational Science as a third mode connecting experiment with theory. Back then such a claim was prophetic, but in the mean time it has become reality. Understanding of NP is no longer possible without scientific computation. One reason for this is, that we have had a steady increase of computational power over a long period of time, and this increase is expected to continue well into the future. This is unprecedented for technological advances. This stems from both improved hardware and algorithms. Keeping up this momentum needs ever new ideas. The upcoming exascale computers illustrate how, after reaching a limit at the level of miniaturization, new programming concepts are needed, to achieve the next level of computational power.

At this point we have three different groups of scientists. We begin with the theoreticians, traditionally trained as mathematicians or theoretical physicists, more recently also as theoretical computer scientists. At the other end are the experimentalists or observers in the field of physics, astrophysics, biology and other natural sciences. In between are computational scientists, who typically have grown out of the theoreticians or out of the experimentalists.

\section{COMPUTATIONAL SCIENCE BREAKING OUT OF ITS THIRD MODE}

In more recent times the increasing importance of computing is now superseding its classical role, namely that of validation of theory, as the interlocutor between theory and experiment. The experiment itself may strongly depend on computation. An example of this dependence is that only computation may handle the huge sets of data accumulated, that data is rendered useful solely through the power of the computer. Another role of computation beyond its traditional role is that the experiment may be calibrated by using computation. For example statistical analysis may guide an experiment. Another example are astronomical observations that are calibrated by numerical simulations leading to an accuracy in observations never before achieved. A final example is how on a desktop computer (having the power today of a supercomputer in the past) models (say traffic models), arising in theory, are computed and thus the qualitative behavior of such models is studied. Here theory begins to depend on computation.

We predict that beyond the traditional role of scientific computation (connecting theory and experiment) and its new important role today in experiments and theory, in the future computation may itself take on an all encompassing role. We predict that computation will become the experiment itself, thus upending the traditional triad NP, experiment and theory. We predict the future ability to compute a myriad of interconnected models, each highly complicated in itself, will play the role of the NP itself. Since this new reality is built up from many theories, a future huge scientific computation will be theory, computation and NP in one.

\section{COMPUTATIONAL PHYSICS AND ITS CHALLENGES}

But lets get back to today. Where does computational physics stand? Whenever the experiment or theory for a given NP involves aspects of physics and in addition there is computation involved, then it belongs to the area of computational physics. This makes computational physics a highly interdisciplinary field. Here we see the playing field of our new journal Frontiers in Computational Physics.

Scientific problems of enormous difficulty are still unsolved. An example of such an extreme challenge is the wish to simulate the evolution of the universe beginning with the microwave radiation background measurement, which depicts the state of the universe soon after the big bang, up to the evolution of our own galaxy, the milky way. Typically such challenging problems involve vastly 
disparate time and length scales. Such problems need advances in theory, computation and experiment. It will be impossible to make inroads in these problems with progress in only one of these areas. Thus one needs to forge synergy between theory, computation and experiment. For many seemingly intractable problems this will be the only possible way to make progress.

Mathematical description of NP has long been the universal language in science. Computation allows for solving ever more complex mathematical models, leading to a reinforcement of the power of the mathematical language. This illustrates how the synergy effect boosts an individual area. But taking advantage of such a boost is a great challenge in itself.

The global weather models by the Intergovernmental Panel on Climate Change (IPCC) are an example of a step beyond scientists in either theory or experiments joined through computation and attempting to communicate say with the help of Frontiers in Computational Physics. Such a huge and complex computational needs a new level of interdisciplinary cooperation. The mathematical description of the IPCC climate model is so complex that it completely eludes progress by mathematical methods alone. The numerical computation of the model will be quite unstable and useless, unless information gathered by careful experimental observations enter this model. The lead scientists in such projects are a new breed, transcending disciplinary boundaries. This development is a welcome countermovement to the specialists that are ever so common. Maybe here lies the ultimate challenge: how to convince specialists to become interdisciplinary, how to train future interdisciplinary scientists without loosing competence in a core area.

\section{THE ROLE OF FRONTIERS IN COMPUTATIONAL PHYSICS}

Becoming more practical, we observe that the tremendous progress in applied mathematics, numerics, and computer science needs to be contrasted with the progress made in computational physics. While physicists are mainly concerned with their original research on physical problems, they cannot necessarily by themselves stay up-to-date with the hottest thing in say numerical analysis. Thus we see an every increasing gap of conceptual results on the one hand, and the implementation and usage of computational tools based on such concepts on the other. Closing this gap, thus adopting new techniques earlier and more frequently, and incorporating them in active computational physics research, seems to us to be one of the great challenges. In particular it is very demanding for a (computational) physicist to identify new developments in applied mathematics, numerics and computer science, and to be able to translate them into research projects. For a computational physicist to identify the potential of a new computational tool, he or she would need to be involved in two distinct research communities, develop metrics and other criteria to judge on the applicability of a particular technique, and finally implement it and validate the results based on his or her expertise in a subfield of physics. Here Frontiers in Computational Physics will attempt to facilitate this dialog.

\section{ACKNOWLEDGMENTS}

The author thanks James Glimm for personal communication on some issues discussed here. The last section and Valdes-Perez's idea were contributed by Kay Hamacher.

\section{REFERENCE}

Valdes-Perez, R. E. (1993). A Scientific Basis for Computational Science. Pittsburgh, PA: Carnegie Mellon University.

Received: 13 May 2013; accepted: 22 May 2013; published online: 26 June 2013.

Citation: Klingenberg C (2013) Grand challenges in computational physics. Front. Physics 1:2. doi: 10.3389/ fphy.2013.00002

This article was submitted to Frontiers in Computational Physics, a specialty of Frontiers in Physics.

Copyright (c) 2013 Klingenberg. This is an open-access article distributed under the terms of the Creative Commons Attribution License, which permits use, distribution and reproduction in other forums, provided the original authors and source are credited and subject to any copyright notices concerning any third-party graphics etc. 